\title{
Otimização da Síntese de Biodiesel de Soja Utilizando Técnicas Quimiométricas.
}

Katherine Lauciene Carlos Oliveira*(Graduando em Ciência e Tecnologia na Universidade Federal Rural do Semi-árido - UFERSA);

Antônio Alex de Lima Silva; (Mestrando em Ciências Naturais na Universidade do Estado do Rio

Grande do Norte - UERN)

Luiz Di Souza; (Prof. Ad. do Dq e do PPGCN na Universidade do Estado do Rio Grande do Norte UERN)

Daniel Freitas Freire Martins; (Docente do curso de Ciência e Tecnologia na Universidade Federal

Rural do Semi-árido - UFERSA)

*E-mail: katherineoliveirako@outlook.com

Resumo:

A maior parte da matriz energética mundial provém do petróleo, carvão e gás natural. Porém essa fonte é limitada e com prazo de esgotamento. Com isso as buscas por fontes alternativas se intensificam cada vez mais, com o propósito de encontrar medidas renováveis, ambientalmente corretas e com baixo custo para suprir total ou parcialmente a dependência do petróleo. Uma fonte alternativa é o biodiesel, que pode ser produzido através de óleo vegetal e gordura animal. 0 presente trabalho teve como objetivo otimizar o processo de produção do biodiesel de óleo de soja, utilizando o planejamento fatorial $2^{3}$ completo com repetição, mostrando as melhores condições e fatores para se obter um maior rendimento na síntese. O biodiesel obtido através da reação de transesterificação foi analisado em diversos parâmetros, tais como: Viscosidade, massa específica, umidade, índice de acidez e saponificação, ponto de fulgor e combustão e sódio e potássio. Com os resultados obtidos, pode-se constatar que o rendimento percentual, índice de saponificação e densidade são os parâmetros mais influenciados pelas condições de síntese do biodiesel, destacandose entre eles o rendimento percentual, já que mesmo influenciados o índice de saponificação e a densidade ainda se encontram dentro das especificações estabelecidas pela ANP. Podendo-se concluir que as condições ideais para produção do biodiesel são com catalisador de $\mathrm{KOH}$, metanol e tempo de reação de 4 horas.

Palavras-chave:

Biodiesel; Óleo de soja; Otimização; Planejamento fatorial; Quimiometria. 


\section{I NTRODUÇÃO}

Sabe-se hoje que a matriz energética mundial provém dos combustíveis fósseis, como o petróleo, carvão e gás natural. Porém, desde a crise energética que se viveu nos anos 70 , provocada pelo desabastecimento e aumento nos preços do petróleo que o mundo inteiro virou as suas atenções e interesses em combustíveis alternativos (KNOTHE et al., 2006 apud GANDOLFI; ARROYO,2007). Em virtude disso, as buscas por fontes alternativas se intensificaram, onde pesquisas são realizadas no mundo inteiro com o proposito de diminuir a dependência do petróleo e seus derivados ou substitui-la totalmente, já que esta fonte energética já apresenta escassez, existindo a possibilidade de esgotamento no futuro (QUINTELA et al., 2007).

Quando levanta-se a questão ambiental, vê-se que os impactos ambientais agravados pela emissão de poluentes de origem fóssil vêm crescendo a cada ano e trazendo danos irreparáveis ao nosso planeta e ao ser humano. Segundo o painel intergovernamental sobre mudanças climáticas a temperatura média do planeta poderá subir de 1,8 a $4^{\circ} \mathrm{C}$ até 2100 , isto devido às emissões de ate $90 \%$ de $\mathrm{CO}_{2}$ derivados de origem fósseis (ROCHA et al., 2007). Mostrando assim, que as fontes alternativas são uma medida precisa.

Dentre as fontes alternativas podemos citar o Biodiesel, produzido através da biomassa dos óleos vegetais, como a soja, mamona, algodão ou através da gordura animal. O Biodiesel segundo Parente (2003), é denominado como um combustível renovável, biodegradável, e ambientalmente correto, sucedâneo ao óleo diesel mineral, constituído de uma mistura de ésteres metílicos ou etílicos de ácidos graxos, obtidos da reação de transesterificação de um triglicerídeo com álcool de cadeia curta, metanol ou etanol, na presença de um catalisador ácido ou básico.

No cenário Brasileiro, país que já conta com $5 \%$ de biodiesel adicionado ao diesel, tem grande potencial para exploração da biomassa. Tendo disponibilidade de terras e clima propício à produção das sementes oleaginosas tanto para fins alimentícios, quanto químico e energético (GONDIM, 2009). Assim, tanto no cenário nacional quanto no internacional é cada vez mais comum a utilização do biodiesel nos postos e motores da população.

Apesar de simples o processo de obtenção de biodiesel, deve-se levar em consideração alguns fatores como, álcool e catalizadores utilizados e tempo de síntese da reação, que podem afetar a qualidade final do produto. Com o intuito de solucionar tais problemas e otimizar a produção, o planejamento fatorial mostra-se uma alternativa eficaz, tendo em vista que tem-se uma grande quantidade de variáveis que podem vir a interferir na reação.

O planejamento fatorial emprega métodos estatísticos e matemáticos que nos permitem selecionar a melhor combinação, ou seja, ela nos mostra quais os melhores níveis e fatores utilizar para otimizar a resposta de um determinado processo, fornecendo o máximo de informação com a análise dos dados

Desta forma, este trabalho teve como objetivo otimizar o processo de produção de biodiesel obtido do óleo de soja, satisfazendo os parâmetros estabelecidos pela ANP, utilizando o planejamento fatorial completo $2^{3}$ com repetição, buscando descobrir a melhor resposta com relação aos parâmetros medidos que dependem dos fatores estudados (Catalisador, álcool e tempo de síntese), verificando se há interação estatisticamente significativa entre eles.

\section{METODOLOGIA}

Para este trabalho realizou-se um planejamento fatorial $2^{3}$, onde utilizou-se 3 fatores e 2 níveis (tabela 01):

Tabela 01: Fatores e níveis utilizados no experimento.

\begin{tabular}{ccc}
\hline Fatores & Nível (-) & Nível (+) \\
\hline Catalisador & $\mathrm{NaOH}$ & $\mathrm{KOH}$ \\
Álcool & Etanol & Metanol \\
Tempo de Síntese & $3 \mathrm{~h}$ & $4 \mathrm{~h}$ \\
\hline
\end{tabular}



02):

Para o planejamento fatorial $2^{3}$ construiu-se uma tabela de coeficientes de contraste (tabela

Tabela 02: Coeficientes de contraste para um planejamento fatorial $2^{3}$.

\begin{tabular}{ccccccc}
\hline $\mathbf{1}$ & $\mathbf{2}$ & $\mathbf{3}$ & $\mathbf{1 2}$ & $\mathbf{1 3}$ & $\mathbf{2 3}$ & $\mathbf{1 2 3}$ \\
\hline-1 & -1 & -1 & 1 & 1 & 1 & -1 \\
1 & -1 & -1 & -1 & -1 & 1 & 1 \\
-1 & 1 & -1 & -1 & 1 & -1 & 1 \\
1 & 1 & -1 & 1 & -1 & -1 & -1 \\
-1 & -1 & 1 & 1 & -1 & -1 & 1 \\
1 & -1 & 1 & -1 & 1 & -1 & -1 \\
-1 & 1 & 1 & -1 & -1 & 1 & -1 \\
1 & 1 & 1 & 1 & 1 & 1 & 1 \\
\hline
\end{tabular}

Onde, a partir da tabela de coeficientes, obteve-se as combinações necessárias para as analises dos ensaios (tabela03):

Tabela 03 - Catalizador, álcool e tempo de síntese utilizada em cada ensaio.

\begin{tabular}{cccc}
\hline Ensaios & Fator $\mathbf{1}$ & Fator $\mathbf{2}$ & Fator $\mathbf{3}$ \\
\hline 1 & $\mathrm{NaOH}$ & $\mathrm{ET}$ & $3 \mathrm{~h}$ \\
2 & $\mathrm{KOH}$ & $\mathrm{ET}$ & $3 \mathrm{~h}$ \\
3 & $\mathrm{NaOH}$ & $\mathrm{MET}$ & $3 \mathrm{~h}$ \\
4 & $\mathrm{KOH}$ & $\mathrm{MET}$ & $3 \mathrm{~h}$ \\
5 & $\mathrm{NaOH}$ & $\mathrm{ET}$ & $4 \mathrm{~h}$ \\
6 & $\mathrm{KOH}$ & $\mathrm{ET}$ & $4 \mathrm{~h}$ \\
7 & $\mathrm{NaOH}$ & $\mathrm{MET}$ & $4 \mathrm{~h}$ \\
8 & $\mathrm{KOH}$ & $\mathrm{MET}$ & $4 \mathrm{~h}$ \\
\hline
\end{tabular}

Para todos os experimentos utilizou-se a razão óleo/álcool 1:6, com concentração do catalisador $2 \%$.

Para a realização dos ensaios houve um sorteio para determinar a ordem dos mesmos, sempre em duplicata. A seguir, descreve-se o método utilizado na síntese do biodiesel:

a. Pesou-se $100 \mathrm{~g}$ de óleo de soja isento de umidade (previamente aquecido por 1 hora a $70^{\circ} \mathrm{C}$ ) em um béquer de $250 \mathrm{ml}$;

b. Em seguida, pesou-se $2 \mathrm{~g}$ de $\mathrm{KOH}$ ou $\mathrm{NaOH}$, de acordo com o planejamento fatorial e transferiu-se para o béquer de $250 \mathrm{ml}$ junto com o óleo;

c. Pesou-se 70g de metanol ou 100g de etanol (Calculada de acordo com a razão óleo/álcool 1:6), para cada ensaio de acordo com o planejamento fatorial e transferiu-se para o béquer de 250 ml contendo o óleo e o catalisador;

d. Manteve-se o sistema em agitação com aproximadamente $400 \mathrm{rpm}$, durante o tempo de cada ensaio previsto no planejamento, 3 ou 4 horas;

e. Em seguida transferiu-se a mistura biodiesel/glicerina para um funil de decantação e deixou-se em repouso durante 24 horas; 
f. Após a visível divisão de fases, separou-se a glicerina e lavou-se o biodiesel com água a $70{ }^{\circ} \mathrm{C}$ para a remoção de impurezas e excesso de catalisador;

g. Transferiu-se o biodiesel para um béquer e colocou-se para secar durante 1 hora na estufa a $70^{\circ} \mathrm{C}$, retirando toda a umidade;

h. Por fim, após resfriado em dessecador, pesou-se o biodiesel e armazenou-se para posteriores analises.

Após o procedimento, calculou-se o rendimento percentual, utilizando a massa do biodiesel e do óleo (Equação1).

$$
\% R=\frac{M_{1} \times 100}{M_{2}}
$$

Onde, $\mathrm{M}_{1}$ é massa do biodiesel e $\mathrm{M}_{2}$ é a massa do óleo.

\subsection{CARACTERI ZAÇÃO DO BIODI ESEL}

- Água e sedimentos: Para determinação de água e sedimentos adotou-se o método ASTM D445. Para a analise utilizou-se $10 \mathrm{~mL}$ da amostra em tubo de escala legível ate $0,1 \mathrm{~mL}$, centrifugada durante 10 min a 800 RCF. Após a centrifugação analisou-se a amostra para constatar se havia presença de água ou sedimentos.

- Índice de acidez: Para a analise do índice de acidez, pesou-se $2 \mathrm{~g}$ da amostra de cada biodiesel em um erlenmeyer, adicionando $25 \mathrm{~mL}$ da solução de éter-álcool (2:1), previamente neutralizada com a solução de hidróxido de sódio 0,1 N. Posteriormente adicionou-se 2 gotas

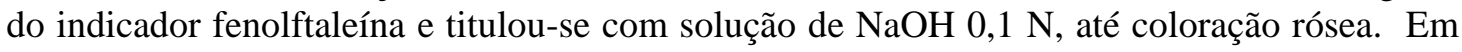
seguida, realizou-se o calculo (Equação 2).

$$
I A=\frac{(V a-V b) \times F C \times 5,61}{M}
$$

\section{(Equação 2)}

Onde: IA é o índice de acidez; Va é o volume (mL) da solução de $\mathrm{NaOH} 0,1$ gastos na solução; $\mathrm{Vb}$ é o volume (mL) da solução de $\mathrm{NaOH}$ 0,1 N gastos na solução branco; FC é o fator de correlação da solução de NaOH; M é a massa (g) da amostra (SANTOS, 2010).

- Viscosidade cinemática: Para analise da viscosidade utilizou-se um viscosímetro adotando a norma ASTM D445. Colocou-se $70 \mathrm{~mL}$ de cada amostra no aparelho deixando aquecer, até a temperatura de $40^{\circ} \mathrm{C}$, controlada através de um termômetro de mercúrio, sendo a temperatura do óleo de aquecimento controlada pelo sistema do aparelho. Após atingir a temperatura, deixou-se o biodiesel escoar, cronometrando o tempo que toda a amostra leva para deixar o aparelho através do furo universal. Em seguida realizou-se o calculo. (Equação 3)

$$
V C C=0,224 x(S S U)-\frac{185}{(S S U)} ; \quad \text { Para } 34 \leq S S U<115
$$

\section{(Equação 3)}

Onde: SSU é o tempo de escoamento em segundos.

- Massa específica $\left(\mathrm{Kg} / \mathbf{m}^{3}\right)$ : A analise da massa específica foi realizada através da técnica de picnometria. Inicialmente, calibrou-se o picnômetro, pesando-o vazio e cheio com água a $29^{\circ} \mathrm{C}$. Com isso, a partir da diferença encontrou-se a massa da água utilizada. Sabendo a densidade da água a $29{ }^{\circ} \mathrm{C}$ encontrou-se o volume através da equação 4 . Desta forma, o volume da água é igual ao volume do picnômetro usado, e assim igual ao do biodiesel. 


$$
d=\frac{m}{v}
$$

Para obtenção da densidade de cada biodiesel, encheu-se o picnômetro calibrado com amostra e pesou-se. Em seguida, pode-se obter a massa do biodiesel através da diferença entre o picnômetro vazio e cheio de cada amostra. Portanto, conhecendo a massa do biodiesel e o volume obteve-se a densidade do biodiesel através da equação 4 .

- Índice de saponificação: Para a determinação do índice de saponificação, pesou-se 2 g de cada amostra em erlenmeyer e adicionou-se $20 \mathrm{~mL}$ da solução alcoólica de hidróxido de potássio 4\%. Após, adaptou-se o erlenmeyer em um condensador de refluxo e deixou-se aquecer até ebulição branda por $30 \mathrm{~min}$. Retira-se a amostra do sistema e adiciona 2 gotas do indicador de fenolftaleína, titula-se a amostra ainda quente com solução de ácido clorídrico 0,5 $\mathrm{N}$ até o total desaparecimento da coloração rósea. Em seguida, realizou-se o calculo (Equação $5)$.

$$
I S=\frac{(V a-V b) \times C t \times 28}{M}
$$

(Equação 5)

Onde: IS é o índice de saponificação de koettstorfer; Va é o volume (mL) do HCL 0,5 N gastos na titulação; Vb é o volume (mL) do HCL 0,5 N gastos na titulação do branco; Ct é a concentração da solução de HCL; M é a massa (mg) da amostra (DANTAS, 2006).

- Teor de umidade: Para esta analise foi adotado o método descrito pela AOCS Bc 2 - 49 (AMERICAM OIL CHEMISTS SOCIETY, 1985). Utilizou-se capsulas de porcelanas previamente lavadas, pesadas e secas na estufa durante 1 hora a $105^{\circ} \mathrm{C}$. Em seguida, pesou-se $5 \mathrm{~g}$ das amostras e colocou-se na estufa durante 1 hora com mesma temperatura do processo anterior. Retirou-se as cápsulas da estufa e deixou-se esfriar ate temperatura ambiente no dessecador, pesou-se e realizou-se os cálculos de acordo com a equação 6.

$$
\% T U=\frac{M_{1}-M_{2}}{M_{1}} \times 100
$$

Onde: $\mathrm{M}_{1}$ é o peso (g) da amostra pesada: $\mathrm{M}_{2}$ é o peso (g) da amostra pesada após o aquecimento.

- Ponto de fulgor e combustão: A analise do ponto de fulgor foi realizada com base na norma analítica ASTM D93, que utiliza aproximadamente $75 \mathrm{~mL}$ da amostra, tendo que chegar ate a marca da cuba do aparelho. Em seguida, coloca-se o termômetro no local indicado no aparelho e liga-se a chama teste. Nota-se o ponto de fulgor quando a fonte de ignição provoca um lampejo na cuba. Já o ponto de combustão que é analisado no mesmo aparelho, nota-se a temperatura apenas quando a amostra permanece em combustão.

- Sódio e potássio: Para análise de sódio e potássio utilizou-se um fotômetro de chama, calibrando o mesmo com uma solução de $\mathrm{Na}^{+}$e $\mathrm{K}^{+}$padrão. Preparou-se então uma solução de ácido clorídrico 0,5 mol/L, sendo a concentração máxima permitida no aparelho. Esta solução foi preparada para solubilizar as amostras e fazer a lavagem do equipamento.

\section{RESULTADOS E DISCUSSÃO}

A tabela a seguir nos mostra os resultados do rendimento percentual obtidos para cada ensaio e suas respectivas medias (Tabela 04). É possível observar claramente que há uma diferença de 
aproximadamente $26 \%$ entre os ensaios que apresentaram o maior e o menor rendimento em massa. Diferença essa bastante significativa, principalmente, quando se trabalha em escala industrial.

Tabela 04 - Resultados do rendimento em percentual da massa dos ensaios

\begin{tabular}{cccc}
\hline Ensaio & R1 (\%) & R2 (\%) & Média (\%) \\
\hline 1 & 69,8601 & 68,7879 & 69,3240 \\
2 & 85,0011 & 88,8911 & 86,9461 \\
3 & 71,9551 & 77,0610 & 74,5080 \\
4 & 93,9968 & 96,7911 & 95,3939 \\
5 & 71,1763 & 76,9074 & 74,0418 \\
6 & 89,6826 & 93,6060 & 91,6443 \\
7 & 90,4581 & 83,8912 & 87,1746 \\
8 & 93,7140 & 92,9162 & 93,3151 \\
\hline
\end{tabular}

Após o calculo do rendimento percentual, calculou-se o erro padrão e o produto do erro pelo $t$ de Student para cada ensaio, assim como os efeitos de $1^{\mathrm{a}}, 2^{\mathrm{a}}$ e $3^{\mathrm{a}}$ ordem (Tabelas 05 e 06).

Tabela 05 - Valores do erro e do produto do erro pelo t de student.

\begin{tabular}{cc}
\hline Erro & $\mathbf{s} * \mathbf{t}$ \\
\hline 1,490382 & 3,427879 \\
\hline
\end{tabular}

Tabela 06 - Efeitos de primeira, segunda e terceira ordem.

\begin{tabular}{ccccccc}
\hline \multicolumn{7}{c}{ Efeitos } \\
\hline $\mathbf{1}$ & $\mathbf{2}$ & $\mathbf{3}$ & $\mathbf{1 2}$ & $\mathbf{1 3}$ & $\mathbf{2 3}$ & $\mathbf{1 2 3}$ \\
\hline 15,56273 & 7,108875 & 5,00095 & $-2,04955$ & $-3,69128$ & 0,292925 & $-3,68145$ \\
\hline
\end{tabular}

Quando se compara o produto do erro pelo t de Student com os efeitos, vê-se que os efeitos significativos são os de primeira ordem 1, 2 e 3, devido o modulo destes efeitos serem maiores que o produto do erro pelo $t$ de Student. Todos os outros efeitos podem ser desconsiderados ou por se apresentarem bem próximos (mesma grandeza) ao produto do erro de Student, ou por serem mais baixos que o mesmo, mostrando que esses efeitos não influenciam significativamente na resposta. Pode-se constatar os mesmos resultados também de acordo com a tabela 07.

Tabela 07 - Relação entre ensaios e média dos rendimentos.

\begin{tabular}{ccccc}
\hline Ensaios & Fator $\mathbf{1}$ & Fator $\mathbf{2}$ & Fator $\mathbf{3}$ & Media (\%) \\
\hline 1 & $\mathrm{NaOH}$ & Etanol & $3 \mathrm{~h}$ & 69,324 \\
2 & $\mathrm{KOH}$ & Etanol & $3 \mathrm{~h}$ & 86,9461 \\
3 & $\mathrm{NaOH}$ & Metanol & $3 \mathrm{~h}$ & 74,50805 \\
4 & $\mathrm{KOH}$ & Metanol & $3 \mathrm{~h}$ & 95,39395 \\
5 & $\mathrm{NaOH}$ & Etanol & $4 \mathrm{~h}$ & 74,04185 \\
6 & $\mathrm{KOH}$ & Etanol & $4 \mathrm{~h}$ & 91,6443 \\
7 & $\mathrm{NaOH}$ & Metanol & $4 \mathrm{~h}$ & 87,17465 \\
8 & $\mathrm{KOH}$ & Metanol & $4 \mathrm{~h}$ & 93,3151 \\
\hline
\end{tabular}


Ao se analisar a tabela 7, ensaios 1 e 2, por exemplo, percebe-se que em relação ao fator catalisador, ao sair do nível (-) para o nível (+), mantendo-se constante os níveis dos outros dois fatores, teve-se um aumento no rendimento percentual do biodiesel de aproximadamente $18 \%$. Com relação ao fator álcool, ensaios 2 e 4, e ao fator tempo de reação, ensaios 3 e 7, por exemplo, ao sair do nível (-) para o nível (+), mantendo-se constante os níveis dos outros fatores, obtém-se o mesmo comportamento apresentado para o fator 1.

Assim como para o rendimento percentual em massa, analisou-se também as características físico-químicas do biodiesel, podendo-se constatar se essas propriedades são influenciadas pelas condições de síntese do biodiesel (Tabela 08). Nesta tabela pode-se visualizar também as especificações estabelecidas pela Agencia Nacional do petróleo, gás natural e biocombustíveis (ANP).

Tabela 08 - Resultado médio da caracterização físico-química do biodiesel, e especificações.

\begin{tabular}{|c|c|c|c|c|c|c|c|c|c|}
\hline \multirow{2}{*}{ Parâmetro } & \multicolumn{8}{|c|}{ Ensaios } & \multirow{2}{*}{$\begin{array}{c}\text { Especificações } \\
\text { da ANP }\end{array}$} \\
\hline & 1 & 2 & 3 & 4 & 5 & 6 & 7 & 8 & \\
\hline Viscosidade & 7,225 & 6,921 & 6,918 & 7,067 & 7,219 & 7,358 & 6,922 & 7,066 & $3,0-6,0 \mathrm{~mm}^{2} / \mathrm{s}$ \\
\hline $\begin{array}{c}\text { Massa } \\
\text { Específica }\end{array}$ & 868,1 & 868,9 & 873,4 & 876,2 & 869,2 & 867,4 & 874,4 & 874,2 & $850-900 \mathrm{~kg} / \mathrm{m}^{3}$ \\
\hline $\begin{array}{c}\text { Índice de } \\
\text { Saponificação }\end{array}$ & 79,69 & 79,97 & 83,16 & 83,53 & 78,52 & 79,57 & 82,76 & 82,17 & NC \\
\hline $\begin{array}{l}\text { Índice de } \\
\text { Acidez }\end{array}$ & 0,271 & 0,271 & 0,272 & 0,272 & 0,407 & 0,272 & 0,271 & 0,272 & $\begin{array}{l}<0,5 \mathrm{mg} \\
\mathrm{KOH} / \mathrm{g}\end{array}$ \\
\hline $\begin{array}{c}\text { Água e } \\
\text { Sedimentos }\end{array}$ & ND & ND & ND & ND & ND & ND & ND & ND & (2) $\mathrm{mg} / \mathrm{kg}$ \\
\hline $\begin{array}{l}\text { Teor de } \\
\text { Umidade }\end{array}$ & 0,785 & 0,462 & 0,304 & 0,414 & 1,109 & 0,992 & 0,268 & 0,306 & NC \\
\hline $\begin{array}{c}\text { Ponto de } \\
\text { Fulgor }\left({ }^{\circ} \mathrm{C}\right)\end{array}$ & 181 & 180 & 185 & 184 & 184 & 183 & 182 & 181 & $>100^{\circ} \mathrm{C}$ \\
\hline $\begin{array}{l}\text { Ponto de } \\
\text { Combustão } \\
\left({ }^{\circ} \mathrm{C}\right)\end{array}$ & 207 & 205 & 205 & 206 & 206 & 207 & 202 & 203 & NC \\
\hline $\begin{array}{l}\text { Sódio + } \\
\text { Potássio }\end{array}$ & ND & ND & ND & ND & ND & ND & ND & ND & $<5 \mathrm{mg} / \mathrm{km}$ \\
\hline
\end{tabular}

$\mathrm{NC}=$ Não consta;

$\mathrm{ND}=$ Não detectado

Contudo, de acordo com o resultado das analises físico-químicas realizadas no biodiesel, notase que os valores estão de acordo com o das especificações estabelecidas, sendo que apenas a viscosidade ultrapassou o valor padrão com apenas um leve acréscimo, podendo ter ocorrido devido a traços remanescentes de glicerina ou óleo, até porque na síntese do biodiesel com o óleo in natura o rendimento em massa não foi de $100 \%$ e a taxa de conversão do óleo em biodiesel não foi determinada ainda (ALVES, 2010). Valores de viscosidade superiores ao especificado na legislação podem causar um atraso na ignição e, consequentemente, influenciar na eficiência da combustão.

Resultados que estão coerentes também com valores apresentados na literatura por (LÔBO, 2009) e (OLIVEIRA, 2012), com apenas a viscosidade apresentando valores um pouco acima do esperado e o índice de saponificação apresentando valores mais baixos do que os apresentados.

Na tabela a seguir (Tabela 09), estão descritos os valores do produto do erro pelo t de Student e seus efeitos de primeira, segunda e terceira ordem para cada parâmetro físico-químico analisado. 
Tabela 09 - Valores dos efeitos e produto do erro pelo t de student.

\begin{tabular}{|c|c|c|c|c|c|c|c|c|}
\hline \multirow{2}{*}{ Parâmetro } & \multicolumn{7}{|c|}{ Efeitos } & \multirow{2}{*}{$\mathbf{s}^{*} \mathbf{t}$} \\
\hline & 1 & 2 & 3 & 12 & 13 & 23 & 123 & \\
\hline Viscosidade & 0,0320 & $-0,1881$ & 0,1086 & 0,1148 & 0,1091 & $-0,107$ & $-0,112$ & 0,1492 \\
\hline $\begin{array}{c}\text { Massa } \\
\text { Específica }\end{array}$ & 0,38 & 6,125 & $-0,362$ & 0,8925 & $-1,41$ & $-0,165$ & $-0,047$ & 0,5537 \\
\hline $\begin{array}{c}\text { Índice de } \\
\text { Saponificação }\end{array}$ & 0,276 & 3,4666 & $-0,833$ & $-0,890$ & $-0,046$ & $-0,048$ & $-0,435$ & 1,0564 \\
\hline $\begin{array}{l}\text { Índice de } \\
\text { Acidez }\end{array}$ & $-0,0338$ & $-0,0338$ & 0,0337 & 0,0339 & $-0,0338$ & $-0,034$ & 0,0339 & 0,0779 \\
\hline $\begin{array}{c}\text { Água e } \\
\text { Sedimentos }\end{array}$ & ND & ND & ND & ND & ND & ND & ND & ND \\
\hline $\begin{array}{l}\text { Teor de } \\
\text { Umidade }\end{array}$ & $-0,0726$ & $-0,5143$ & 0,1776 & 0,1465 & 0,0336 & $-0,2495$ & $-0,0696$ & 0,4141 \\
\hline $\begin{array}{l}\text { Ponto de } \\
\text { Fulgor }\end{array}$ & $-0,875$ & 0,625 & $-0,125$ & 0,125 & $-0,125$ & $-3,125$ & $-0,125$ & 3,9524 \\
\hline $\begin{array}{l}\text { Ponto de } \\
\text { Combustão }\end{array}$ & 0,125 & $-2,625$ & $-1,375$ & 0,625 & 0,875 & $-1,875$ & $-0,625$ & 3,5562 \\
\hline $\begin{array}{l}\text { Sódio + } \\
\text { Potássio }\end{array}$ & ND & ND & ND & ND & ND & ND & ND & ND \\
\hline
\end{tabular}

Ao se analisar os dados, pode-se perceber que os parâmetros da viscosidade, índice de acidez, água e sedimento, teor de umidade, ponto de fulgor e combustão e sódio e potássio, não são afetados pelas modificações nas condições de síntese do biodiesel, já que os mesmos não apresentaram efeitos significativos na resposta. Todos os efeitos se apresentam próximos (mesma grandeza) ou menores que o produto do erro pelo t de Student. Já quando analisa-se os resultados dos parâmetros da massa especifica e índice de saponificação, percebe-se que o efeito de primeira ordem 2, em ambos, são superiores que o valor do produto do erro pelo t de Student, mostrando que os mesmo tem valor significativo para a resposta final. Pode-se analisá-los de outra forma conforme a tabela 10 e 11.

Tabela 10 - Resultados das médias da massa específica dos ensaios.

\begin{tabular}{|c|c|c|c|c|}
\hline Ensaios & Fator 1 & Fator 2 & Fator 3 & $\begin{array}{c}\text { Media } \\
\left(\mathrm{kg} / \mathrm{m}^{3}\right)\end{array}$ \\
\hline 1 & $\mathrm{NaOH}$ & Etanol & $3 \mathrm{~h}$ & 868,1 \\
\hline 2 & $\mathrm{KOH}$ & Etanol & $3 \mathrm{~h}$ & 868.9 \\
\hline 3 & $\mathrm{NaOH}$ & Metanol & $3 \mathrm{~h}$ & 873,4 \\
\hline 4 & $\mathrm{KOH}$ & Metanol & $3 \mathrm{~h}$ & 876,2 \\
\hline 5 & $\mathrm{NaOH}$ & Etanol & $4 \mathrm{~h}$ & 869,2 \\
\hline 6 & $\mathrm{KOH}$ & Etanol & $4 \mathrm{~h}$ & 867,4 \\
\hline 7 & $\mathrm{NaOH}$ & Metanol & $4 \mathrm{~h}$ & 874,4 \\
\hline 8 & $\mathrm{KOH}$ & Metanol & $4 \mathrm{~h}$ & 874,2 \\
\hline
\end{tabular}


Tabela 11 - Resultado das médias do índice de saponificação dos ensaios.

\begin{tabular}{ccccc}
\hline Ensaios & Fator 1 & Fator $\mathbf{2}$ & Fator $\mathbf{3}$ & $\begin{array}{c}\text { Media } \\
\left.\mathbf{( K g} / \mathbf{m}^{3}\right)\end{array}$ \\
\hline 1 & $\mathrm{NaOH}$ & Etanol & $3 \mathrm{~h}$ & 79,69 \\
2 & $\mathrm{KOH}$ & Etanol & $3 \mathrm{~h}$ & 79,97 \\
3 & $\mathrm{NaOH}$ & Metanol & $3 \mathrm{~h}$ & 83,16 \\
4 & $\mathrm{KOH}$ & Metanol & $3 \mathrm{~h}$ & 83,53 \\
5 & $\mathrm{NaOH}$ & Etanol & $4 \mathrm{~h}$ & 78,52 \\
6 & $\mathrm{KOH}$ & Etanol & $4 \mathrm{~h}$ & 79,57 \\
7 & $\mathrm{NaOH}$ & Metanol & $4 \mathrm{~h}$ & 82,76 \\
8 & $\mathrm{KOH}$ & Metanol & $4 \mathrm{~h}$ & 82,17 \\
\hline
\end{tabular}

Ao analisar-se a tabela da massa específica, tem-se que em relação ao fator 2 (álcool), ensaios 2 e 4, por exemplo, ao sair do nível (-) para o nível (+) tem-se um aumento da densidade do biodiesel, podendo estar relacionada com a sua estrutura molecular. Para os resultados do índice de saponificação, tem-se o mesmo comportamento também em relação ao fator 2 (álcool), ao sair do nível (-) para o nível (+), há um aumento deste índice.

\section{CONCLUSÃO}

De acordo com os parâmetros analisados e resultados obtidos, pode-se constatar que o rendimento percentual em massa é o parâmetro que mais sofre alteração, sendo afetado diretamente pelas condições de síntese do biodiesel. Já a densidade como o índice de saponificação que também tem valores significativos, apesar de serem influenciadas pelas condições de síntese, estão dentro das normas da Agencia Nacional do petróleo, gás natural e biocombustíveis (ANP). Portanto, pode-se concluir a partir das respostas obtidas que as condições ideais para síntese do biodiesel são: o catalizador de Hidróxido de potássio $(\mathrm{KOH})$, Álcool Metanol $\left(\mathrm{CH}_{4} \mathrm{O}\right)$ e tempo de síntese de 4 horas. Contudo, o biodiesel com catalisador de Hidróxido de potássio $(\mathrm{KOH})$, álcool Etanol $\left(\mathrm{C}_{2} \mathrm{H}_{6} \mathrm{O}\right)$ e tempo de reação de $4 \mathrm{hrs}$, apresenta-se como um biodiesel com um rendimento percentual mais baixo, mais satisfatório, além de conter uma vantagem em relação ao álcool utilizado já que o etanol é renovável e não apresenta riscos a saúde e é produzido abundantemente no Brasil.

\section{Optimization of Soybean Biodiesel Synthesis Using Chemometric Techniques.}

Albstract: The majority of global energy comes from oil, coal and natural gas. But this source is limited and term exhaustion. Thus the search for alternative sources intensifies increasingly, with the purpose of finding renewable measures, environmentally sound and cost to meet all or part of the dependence on oil. An alternative source is biodiesel, which can be produced by vegetable oil and animal fat. This study aimed to optimize the soybean oil biodiesel production process using the full 23 factorial design with repetition, showing the best conditions and factors to obtain a higher yield in the synthesis. The biodiesel obtained from the transesterification reaction was analyzed on various parameters, such as viscosity, density, moisture, acidity and saponification index, flash point and combustion and sodium and potassium. With the results obtained, it can be seen that the percent yield, saponification number and density are the most influenced parameters for biodiesel synthesis conditions, especially including the percent yield, since even influenced the saponification index and density are still within the specifications set by the ANP. It can be concluded that the optimum conditions for production of biodiesel are $\mathrm{KOH}$ catalyst, methanol and ethanol time of 4 hours reaction 
Keywords: Biodiesel; Soy oil; optimize ; Factorial design; chemometrics.

\section{Referências Bibliográficas}

NETO, B. B.; SCARMINIO, I. S.; BRUNS, R. E. Como fazer experimentos. UNICAMP. Campinas/SP, 2007.

OLIVEIRA, I. K. Aplicação de quimiometria e espectroscopia no infravermelho no controle de qualidade de biodiesel e mistura biodiesel/diesel. Dissertação (Mestrado em química analítica) UNICAMP. p. 110, 2008.

LÔBO, I. P.; FERREIRA, S. L. C. BIODIESEL: Parâmetros de qualidade e métodos analíticos. Química Nova, Vol. 32, No 6, 1596-1608, 2009.

FERREIRA, M. M. C. et al. Quimiometria I: calibração multivariada, um tutorial. Química Nova, v. 22, n. 5, p.724-731, 1999.

SANTOS, A. G. D. Avaliação da estabilidade térmica e oxidativa dos biodieseis de algodão, girassol, dendê e sebo bovino. Dissertação (Mestrado em química) - UFRN. Natal/RN, 2010.

AMORIN, M. C. Fonte de energia alternativa: Um estudo sobre a percepção ambiental, social e econômica do biodiesel com docentes do centro de tecnologia do gás da cidade de Natal-RN. Dissertação (Mestrado em ciências em engenharia de produção) - UFRN. p.111, 2009.

KUCEK, K. T. Otimização da transferência etílica do óleo de soja em meio alcalino. Dissertação (Mestrado em química orgânica). Curitiba, 2004.

ARAÚJO, L. C. L. F. Síntese, caracterização e aplicação de LaSBA-15 e como catalisador para obtenção de biodiesel de soja via rota etílica. Dissertação (Mestrado em Pesquisa e Desenvolvimento em Ciência e Engenharia de Petróleo) - UFRN. Natal, 2011.

CARVALHO, R. H. R. Avaliação da eficiência de catalisadores comerciais no obtenção de biodiesel de algodão (Gossipium hisutum L.). Dissertação (Mestrado em Pesquisa e Desenvolvimento de Tecnologias Regionais) - UFRN. Natal/RN. 2009.

NETO, B. B.; SCARMINIO, I. S.; BRUNS, R. E. 25 anos de quimiometria no brasil. Quim. Nova, Vol. 29, No. 6, 1401-1406, 2006.

KOHLHELPP, G. Análise as situação da produção de etanol e biodiesel no brasil. Estud. av. vol.24 no.68 São Paulo, 2010.

OLIVEIRA, D. S.; FONSECA, D. S.; FARIAS, P. N.; BEZERRA, V. S.; et al. Obtenção do biodiesel através da transesterificação do óleo de moringa oleífera lam. HOLOS, ISSN 1087 -1600. 2012.

FERRARI, R. A.; OLIVEIRA, V. S.; SCABIO, A. Biodiesel de soja - Taxa de conversão em ésteres etílicos, caracterização físicoquímica e consumo em gerador de energia. Química Nova, Vol. 28, No. 1, 19-23, 2005. 